\title{
THE BOREL STRUCTURE OF ITERATES OF CONTINUOUS FUNCTIONS
}

\author{
by PAUL D. HUMKE* and M. LACZKOVICH*
}

(Received 11 th October 1988)

\section{Notation}

Let $\mathscr{C}[0,1]$ be the Banach space of continuous functions defined on $[0,1]$ and let $\mathscr{C}$ be the set of functions $f \in \mathscr{C}[0,1]$ mapping $[0,1]$ into itself. If $f \in \mathscr{C}, f^{k}$ will denote the $k$ th iterate of $f$ and we put $\mathscr{C}^{k}=\left\{f^{k}: f \in \mathscr{C}\right\}$. The set of increasing ( $\equiv$ nondecreasing) and decreasing ( $\equiv$ nonincreasing) functions in $\mathscr{C}$ will be denoted by $\mathscr{I}$ and $\mathscr{D}$, respectively. If a function $f$ is defined on an interval $I$, we let $C(f)$ denote the set of points at which $f$ is locally constant, i.e.

$$
C(f)=\{x \in I: \text { there is a } \delta>0 \text { such that } f \text { is constant on }(x-\delta, x+\delta) \cap I\} \text {. }
$$

We let $\mathbb{N}$ denote the set of positive integers and $\mathbb{N}^{\mathbb{N}}$ denote the Baire space of sequences of positive integers.

\section{Increasing iterates}

In this section we prove that the sets $\mathscr{C}^{k}$ and $\mathscr{C}^{k} \cap \mathscr{I}$ are analytic and non-Borel subsets of $\mathscr{C}[0,1]$ for every $k \geqq 2$. The fact that $\mathscr{C}^{k}$ is analytic follows directly from the continuity of the mapping $f \mapsto f^{k}(f \in \mathscr{C})$. As $\mathscr{I}$ is closed in $\mathscr{C}[0,1]$, the set $\mathscr{C}^{k} \cap \mathscr{I}$ is also analytic. The goal of the next series of lemmas is to show that for each $k \geqq 2$ and for each Borel subset $B \subset \mathbb{N}^{N}$ there is a continuous map $F: \mathbb{N}^{N} \rightarrow \mathscr{I}$ such that $F^{-1}\left(\mathscr{C}^{k}\right)=$ $B$. From this it easily follows that neither of the sets $\mathscr{C}^{k}$ nor $\mathscr{C}^{k} \cap \mathscr{I}$ is Borel. Indeed suppose $\mathscr{C}^{k}$ or $\mathscr{C}^{k} \cap \mathscr{I}$ is Borel and is of Borel class $\alpha<\omega_{1}$. We can choose a Borel set $B \subset \mathbb{N}^{N}$ of class higher than $\alpha$ and construct a map $F$ as above. Since $F$ is continuous and maps into $\mathscr{I}, F^{-1}\left(\mathscr{C}^{k}\right)=F^{-1}\left(\mathscr{C}^{k} \cap \mathscr{I}\right)=B$ is of class $\alpha$ which is contrary to the choice of $B$.

In order to construct this mapping, $F$, we introduce the following subclasses of $\mathscr{I}$. For any choice of numbers $0<a<b<c<1$ we let $\mathscr{N}_{a b c}$ denote the set of functions $f \in \mathscr{I}$ satisfying the following conditions.

1. $f(0)=0$ and $f(1)=1$.

*The work of both authors was supported in part by the Fulbright foundation. 
2. $f(a)=b$ and $f(b)=c$.

3. $f$ is linear on each of the intervals $[0, a]$ and $[b, 1]$.

Our initial aim is to characterize the functions belonging to $\mathscr{N}_{a b c} \cap \mathscr{C}^{k}$ in terms of the set $C(f)$. Throughout the remainder of this section we consider the numbers $a, b$, and $c$ fixed and simply use $\mathcal{N}$ to denote $\mathcal{N}_{a b c}$.

We begin with the following simple lemma whose proof is omitted.

Lemma 1. Let $h_{1}$ and $h_{2}$ be increasing and continuous functions defined on the closed interval $[x, y]$ such that $C\left(h_{1}\right)=C\left(h_{2}\right)$. Then there is a strictly increasing continuous function $j$ defined on $\left[h_{1}(x), h_{1}(y)\right]$ such that $h_{2}=j \circ h_{1}$.

Lemma 2. Let $f \in \mathscr{N} \cap \mathscr{C}^{k}, k \geqq 2$. Then there exists a $g \in \mathscr{I}$ and points $a=x_{0}<x_{1}<$ $\cdots<x_{k}=b$ such that $f=g^{k}, g(0)=0$ and $g(1)=1, g\left(x_{i}\right)=x_{i+1}, i=0,1, \ldots, k-1$ and $g$ is strictly increasing on each of the intervals $\left[0, x_{k-1}\right]$ and $[b, 1]$.

Proof. As $f \in \mathscr{C}^{k}$, there is a $g \in \mathscr{C}$ such that $f=g^{k}$. Since $f$ has no fixed point in $(0,1)$, neither does $g$. Consequently, either $g(x)<x$ holds for every $x \in(0,1)$ or $g(x)>x$ holds for every $x \in(0,1)$. The former entails that $f(x)=g^{k}(x) \leqq g^{k-1}(x) \leqq \cdots \leqq g(x)<x$ for every $x \in(0,1)$ which is not the case. Then $g(x)>x$ for each $x \in(0,1)$ and this fact implies that $g(1)=1$; as $f(0)=0$ we also deduce that $g(0)=0$. Also, as $f(x)<1$ for $x<1$ it follows that $g(x)<1$ for $x<1$. We have

$$
x<g(x)<\cdots<g^{k}(x)=f(x) \text { for } x \in(0,1) .
$$

Define $x_{i}=g^{i}(a), i=0,1, \ldots, k$. By (2.1) and the fact that $f(a)=b$ we have $a=$ $x_{0}<x_{1}<\cdots<x_{k}=b$. Now, $g^{k-1}(0)=0$ and $g^{k-1}(a)=x_{k-1}$ so that $\left[0, x_{k-1}\right] \subset g^{k-1}([0, a])$. But $f(x)=g^{k}(x)=g\left(g^{k-1}(x)\right)$ and $f$ is injective on $[0, a]$. Hence, $g$ is injective on $\left[0, x_{k-1}\right]$ and as $g(0)=0, g$ is strictly increasing there. Similarly, as $f=g^{k}$ is injective on $[b, 1], g$ is strictly increasing on $[b, 1]$. What remains is to prove that $g$ is increasing on $\left[x_{k-1}, b\right]$. As $g^{k-1}(a)=x_{k-1}$ and $g^{k-1}\left(x_{1}\right)=b$ it follows that $g^{k-1}\left(\left[a, x_{1}\right]\right)=\left[x_{k-1}, b\right]$. But then the result follows by noting that $g^{k-1}$ is strictly increasing on $\left[a, x_{1}\right]$ and $f=g\left(g^{k-1}\right)$ is increasing on $\left[a, x_{1}\right]$.

Lemma 3. For every $f \in \mathcal{N}$ and $k \geqq 2, f \in \mathscr{C}^{k}$ if and only if there are points $a=x_{0}<x_{1}<\cdots<x_{k}=b$ and a function $\phi$ defined on $\left[x_{0}, x_{k-1}\right]$ such that for each $i=1,2, \ldots, k-1, \phi$ is an increasing homeomorphism mapping $\left[x_{i-1}, x_{i}\right]$ onto $\left[x_{i}, x_{i+1}\right]$ satisfying

$$
\phi\left(C\left(f \mid\left[x_{i-1}, x_{i}\right]\right)\right)=C\left(f \mid\left[x_{i}, x_{i+1}\right]\right) .
$$

Proof. If $f \in \mathcal{N} \cap \mathscr{C}^{k}$ then there are points $a=x_{0}<x_{1}<\cdots<x_{k}=b$ and a function $g \in \mathscr{C}$ which satisfy the conclusion of Lemma 2 . Let $\phi=g \mid\left[x_{0}, x_{k-1}\right]$. It follows directly 
from Lemma 2 that for each $i=1,2, \ldots, k-1, \phi$ is an increasing homeomorphism mapping $\left[x_{i-1}, x_{i}\right]$ onto $\left[x_{i}, x_{i+1}\right]$. As $g$ is strictly increasing on each of the intervals $\left[x_{i-1}, x_{i}\right] i=1,2, \ldots, k-1$ and on $[b, 1]$ we have

$$
\phi\left(C\left(f \mid\left[x_{i-1}, x_{i}\right]\right)\right)=\phi\left(C\left(g \circ f \mid\left[x_{i-1}, x_{i}\right]\right)\right)=\phi\left(C\left(f \circ \phi \mid\left[x_{i-1}, x_{i}\right]\right)\right)=C\left(f \mid\left[x_{i}, x_{i+1}\right]\right) .
$$

This completes the proof of the necessity and we now turn to the sufficiency proof.

Suppose that the numbers $x_{i}, i=0,1, \ldots, k$ and the function $\phi$ are given and satisfy the conditions of the lemma. We prove that $\phi$ can be extended to a continuous function $g$ defined on $[0,1]$ such that $f=g^{k}$. First note that $f\left(x_{i-1}\right)<f\left(x_{i}\right)(i=1, \ldots, k)$. Indeed, if $f\left(x_{i-1}\right)=f\left(x_{i}\right)$ then $C\left(f \mid\left[x_{i-1}, x_{i}\right]\right)=\left[x_{i-1}, x_{i}\right]$. This implies, by (3.1) that $f$ is constant on the entire interval $[a, b]$. This, of course, contradicts the fact that $f(a)=b<c=f(b)$.

Next, we extend the sequence $\left\{x_{0}, x_{1}, \ldots, x_{k}\right\}$ by defining $x_{n}=f\left(x_{n-k}\right)$ for $n>k$ and $x_{n}=f^{-1}\left(x_{n+k}\right)$ for $n<0$. Since $f$ is strictly increasing on each of the intervals $[0, a]$ and $[b, 1]$, and $x_{k}<x_{k+1}<\cdots<x_{2 k-1}$ (our prior remark) it is easy to verify that $x_{n}<x_{n+1}$ for every integer $n$. If $v=\lim _{n \rightarrow \infty} x_{n}$ then $f(v)=v$ and as $v>0, v=1$. Similarly, $\lim _{n \rightarrow \infty} x_{-n}=0$.

We inductively define a function $\phi_{n}$ on the interval $\left[x_{n-1}, x_{n}\right]$ such that

$A_{n} . \quad \phi_{n}$ is increasing and continuous on $\left[x_{n-1}, x_{n}\right]$.

$B_{n} . \phi_{n}$ maps $\left[x_{n-1}, x_{n}\right]$ onto $\left[x_{n}, x_{n+1}\right]$.

$C_{n}$. If $n \neq k$, then $\phi_{n}$ is strictly increasing.

We begin by defining $\phi_{n}=\phi \mid\left[x_{n-1}, x_{n}\right]$ for $n=1,2, \ldots, k-1$. By hypothesis, $A_{n}, B_{n}$, and $C_{n}$ are true for these $n$. Next we define $\phi_{k}=f \circ \phi_{1}^{-1} \circ \phi_{2}^{-1} \circ \cdots \circ \phi_{k-1}^{-1}$ and note that $A_{k}$ and $B_{k}$ are satisfied. Suppose now that $n \geqq 0$ and that for each $i=1,2, \ldots, n+k, \phi_{i}$ has been defined and satisfies $A_{i}, B_{i}$, and $C_{i}$. We prove that

$$
C\left(\phi_{n+k} \circ \phi_{n+k-1} \circ \cdots \circ \phi_{n+2}\right)=C\left(f \mid\left[x_{n+1}, x_{n+2}\right]\right) .
$$

There are two cases. First suppose that $n \leqq k-2$. Then, as the functions $\phi_{k+1}, \phi_{k+2}, \ldots, \phi_{k+n}$ are strictly increasing (property $\left.C_{i}\right)$, the left hand side of (3.2) reduces to $C\left(\phi_{k} \circ \phi_{k-1} \circ \cdots \circ \phi_{n+2}\right)$. Using (3.1) and the definition of $\phi_{k}$ it is easy to check that $C\left(\phi_{k} \circ \phi_{k-1} \circ \cdots \circ \phi_{n+2}\right)=C\left(f \mid\left[x_{n+1}, x_{n+2}\right]\right)$. If $n>k-2$ then all of the functions extant in (3.2) are strictly increasing so that both sides of (3.2) are empty. We apply Lemma 1 with $h_{1}=\phi_{n+k} \circ \phi_{n+k-1} \circ \cdots \circ \phi_{n+2}, h_{2}=f \mid\left[x_{n+1}, x_{n+2}\right]$. Thus we obtain a strictly increasing continuous function, $\phi_{n+k+1}$, defined on $h_{1}\left(\left[x_{n+1}, x_{n+2}\right]\right)=$ $\left[x_{n+k}, x_{n+k+1}\right]$ such that

$$
\phi_{n+k+1} \circ \phi_{n+k} \circ \cdots \circ \phi_{n+2}=f \mid\left[x_{n+1}, x_{n+2}\right] .
$$

Again, conditions $A_{n+k+1}, B_{n+k+1}$, and $C_{n+k+1}$ are satisfied. Hence $\phi_{n}$ has been defined for every $n>0$ and we now turn to the case when $n \leqq 0$.

Suppose $n \leqq 0$ and that for each $i>n, \phi_{i}$ has been defined and satisfies the conditions $A_{i}, B_{i}$, and $C_{i}$. We put 


$$
\phi_{n}=\phi_{n+1}^{-1} \circ \phi_{n+2}^{-1} \circ \cdots \circ \phi_{n+k-1}^{-1} \circ\left(f \mid\left[x_{n-1}, x_{n}\right]\right) \text {. }
$$

As in the previous cases, $A_{n}, B_{n}$, and $C_{n}$ are transparent. In this way, $\phi_{n}$ has been defined for every integer $n$ and we define

$$
g(x)= \begin{cases}\phi_{n}(x) & \text { if } x \in\left[x_{n-1}, x_{n}\right], n \in \mathbb{Z} \\ 0 & \text { if } x=0 \text { and } 1 \text { if } x=1\end{cases}
$$

It follows from the conditions $A_{n}$ and $B_{n}$ that $g$ is increasing and continuous on $[0,1]$. Now, if $x \in(0,1)$ then there is an integer $n$ such that $x \in\left[x_{n-1}, x_{n}\right]$. If $n \leqq 0$ then $f(x)=g^{k}(x)$ by (3.4); if $n \geqq 2$ then $f(x)=g^{k}(x)$ by (3.3). Then sole remaining case is that when $n=1$ and the fact that $f(x)=g^{k}(x)$ for $x \in\left[x_{0}, x_{1}\right]$ follows from the definition of $\phi_{k}$. The proof of Lemma 3 is completed by noting that 0 and 1 are fixed points of both $g$ and $f$.

A family of subsets of $\mathbb{R},\left\{I_{\gamma}: \gamma \in \Gamma\right\}$, is said to be discrete if there is a family of pairwise disjoint open sets $\left\{U_{\gamma}: \gamma \in \Gamma\right\}$ such that $\bar{I}_{\gamma} \subseteq U_{\gamma}(\bar{A} \equiv A$ closure) for every $\gamma \in \Gamma$. A family of pairwise disjoint intervals will be considered ordered according to the usual ordering of $\mathbb{R}$.

Lemma 4. Let $\beta$ be an infinite countable ordinal, $\varepsilon>0$ and $k \geqq 2$. Suppose that $\left\{I_{\alpha}: \alpha<\beta k\right\}$ is a discrete set of open intervals contained in $(a+\varepsilon, b-\varepsilon)$ such that $I_{\alpha}<I_{\gamma}$ for $\alpha<\gamma<\beta k$. Then there are points $a=x_{0}<x_{1}<\cdots<x_{k}=b$ and a homeomorphism $\phi:\left[x_{0}, x_{k-1}\right] \rightarrow\left[x_{1}, x_{k}\right]$ such that $I_{\beta i+\alpha} \subset\left[x_{i}, x_{i+1}\right](i=0, \ldots, k-1, \alpha<\beta), \phi$ maps $\left[x_{i-1}, x_{i}\right]$ onto $\left[x_{i}, x_{i+1}\right]$ and $I_{\beta(i-1)+\alpha}$ onto $I_{\beta i+\alpha}$ for each $i=1,2, \ldots, k-1$ and each $\alpha<\beta$.

Proof. For every $\alpha<\beta k$ let $I_{\alpha}=\left(u_{\alpha}, v_{\alpha}\right)$ and define $w_{i}=\lim _{\alpha \rightarrow \beta i} v_{\alpha}$ for each $i=1,2, \ldots, k$. As $\left\{I_{\alpha}: \alpha<\beta k\right\}$ is discrete, $w_{i}<u_{\beta i}$ for $i \leqq k-1$ and $w_{k} \leqq b-\varepsilon$. Let $x_{0}=a, x_{i}=\left(w_{i}+u_{\beta i}\right) / 2$ $(i=1,2, \ldots, k-1)$ and $x_{k}=b$. Then define $\phi\left(x_{k-1}\right)=b ; \phi\left(x_{i}\right)=x_{i+1}, \phi\left(u_{\beta i+\alpha}\right)=u_{\beta(i+1)+\alpha}$ and $\phi\left(v_{\beta i+\alpha}\right)=v_{\beta(i+1)+\alpha}$ for $i=0,1, \ldots, k-2$ and $\alpha<\beta$. As $\phi$ is strictly increasing on its domain and $\left\{I_{\alpha}: \alpha<\beta k\right\}$ is discrete, $\phi$ can be extended to a strictly increasing continuous function defined on the closure of its domain. We further extend $\phi$ to the entire interval $\left[x_{0}, x_{k-1}\right]$ by defining the extension to be linear on each component of the complement of this closure. This completes the proof of Lemma 4.

Let $E \subseteq[0,1] \times[0,1]$ and $x, y \in[0,1]$. We denote the vertical and horizontal sections of $E$ by $E_{x}=\{y:(x, y) \in E\}$ and $E^{y}=\{x:(x, y) \in E\}$. Now let $\left\{J_{\gamma}: \gamma \in \Gamma\right\}$ be a discrete family of open intervals in $[a, b]$ and let $K=[a, b] \backslash \bigcup_{\gamma \in \Gamma} J_{\gamma}$. Then each portion of $K$ has positive Lebesgue measure. Let $\left\{I_{\gamma}: \gamma \in \Gamma\right\}$ be a family of subintervals of $[0,1]$ with rational endpoints, and define $G=\bigcup_{\gamma \in \Gamma}\left(J_{\gamma} \times I_{\gamma}\right)$. We define a map $T:[0,1] \rightarrow \mathscr{C}[a, b]$ as follows ( $\lambda \equiv$ Lebesgue measure).

$$
T(y)(x)=\frac{\lambda\left([a, x] \backslash G^{y}\right)}{\lambda\left([a, b] \backslash G^{y}\right)}, \quad y \in[0,1] \quad \text { and } \quad x \in[a, b]
$$


Lemma 5. The map $T$ defined above has the following properties.

1. $T(y)$ is increasing and continuous for every $y \in[0,1]$.

2. $T(y)(a)=0$ and $T(y)(b)=1$ for every $y \in[0,1]$.

3. $C(T(y))=G^{y}$ for every $y \in[0,1]$.

4. $T$, as a map from $[0,1]$ into $\mathscr{C}[a, b]$, is continuous at each irrational $y \in[0,1]$.

Proof. Statements 1 and 2 are obvious and 3 follows from the fact that every portion of $[a, b] \backslash \bigcup_{y \in \Gamma} J_{\gamma}$ has positive Lebesgue measure. To prove 4 , first note that $\Gamma$ is countable. Let $y_{0} \in[0,1]$ be irrational, and let $\left\{\gamma_{1}, \gamma_{2}, \ldots, \gamma_{n}\right\} \subset \Gamma$ be an arbitrary finite set of indices. As the endpoints of the $I_{\gamma}$ are rational, there is a $\delta>0$ such that if $y \in\left(y_{0}-\delta, y_{0}+\delta\right)$ then $y \in I_{\gamma_{j}}$ if and only if $y_{0} \in I_{\gamma_{j}}$ for $j=1,2, \ldots, n$. The continuity of $T$ at $y_{0}$ easily follows from this observation.

Lemma 6. If $B \subset[0,1]$ is Borel, then there is a set $M \subseteq[0,1] \times[0,1]$ consisting of $a$ countable union of vertical line segments with rational endpoints and a countable ordinal $\beta$ such that:

1. If $y \in B$, then $M^{y}$ is well ordered with ordertype less than $\beta$;

2. if $y \notin B$, then $M^{y}$ is not well ordered, but every decreasing sequence in $M^{y}$ converges to the same real number.

Proof. For $i \in \mathbb{N}$ and $\sigma \in \mathbb{N}^{N}$ we denote the restriction of $\sigma$ to its first $i$ coordinates by $\sigma \mid i$. The desired set $M$ is a Lusin sieve for $\mathbb{R} \backslash B$ and the special characteristics of $M$ are derived from the fact that $B$ is Borel. Specifically, there is a set of closed intervals with rational endpoints, $\left\{I_{\tau}: \tau \in \mathbb{N}^{i}, i=1,2, \ldots\right\}$ satisfying the following conditions:

(i) $\mathbb{R} \backslash B=\bigcup_{\sigma \in \mathbb{N}^{N}} \bigcap_{i=1}^{\infty} I_{\sigma \mid i}$.

(ii) If $n>m$ and $\sigma \in \mathbb{N}^{\mathbb{N}}$, then $I_{\sigma \mid n} \subset I_{\sigma \mid m}$.

(iii) For every $y \in \mathbb{R} \backslash B$ there is a unique $\sigma \in \mathbb{N}^{N}$ such that $\{y\}=\bigcap_{i=1}^{\infty} I_{\sigma \mid i}$.

We form a Lusin sieve for $\mathbb{R} \backslash B$ by assigning to each finite sequence of natural numbers $\tau=\left(n_{1}, n_{2}, \ldots, n_{i}\right)$ the binary fraction $x_{\tau}=1-2^{-n_{1}}-\cdots-2^{-n_{1}-\cdots-n_{i}}$ and the closed interval $I_{\tau}$. The set $M$ is defined as $M=\cup\left(\left\{x_{\tau}\right\} \times I_{z}\right)$ where the union is taken over all finite sequences of natural numbers. It follows directly from the definition of the sieve that $M^{y}$ is well ordered if and only if $y \in B$. The fact that there is a countable ordinal $\beta$ bounding the ordinals of the sections $M^{y}, y \in B$ is the substance of Corollary $5 a$ of Section 39, VIII in [1]. Finally, suppose $y \in \mathbb{R} \backslash B$. Then there is a unique sequence $\sigma=\left(n_{1}, n_{2}, \ldots\right)$ such that $y \in \bigcap_{i=1}^{\infty} I_{\sigma \mid i}$. We prove that every decreasing sequence in $M^{y}$ converges to the point $1-x$ where $x=2^{-n_{1}}+2^{-n_{1}-n_{2}}+\ldots$.

Suppose $\left\{x_{\tau_{i}}\right\}$ is an increasing sequence of binary fractions such that $1-x_{\tau_{i}} \in M^{y}$ for every $i$. For each $j$ we denote the $j$ th coordinate of $\tau_{i}$ by $\tau_{i}(j)$. It is easily verified that for each fixed $j$, the sequence $\left\{\tau_{i}(j): i=1,2, \ldots\right\}$ is eventually decreasing and hence, is eventually stationary at a natural number which we denote by $\tau(j)$. If $\tau=(\tau(1), \tau(2), \ldots)$ 
then $y \in \bigcap_{i=1}^{\infty} I_{\tau \mid i}$ and as $\sigma$ is unique, $\sigma=\tau$. Hence, $\left\{x_{\tau_{i}}: i=1,2, \ldots\right\}$ converges to $x=2^{-n_{1}}+2^{-n_{1}-n_{2}}+\ldots$

Lemma 7. For every Borel set $B \subset \mathbb{N}^{N}$ and every $k \geqq 2$ there is a continuous function $F: \mathbb{N}^{N} \rightarrow \mathcal{N}$ such that $F(y) \in \mathscr{C}^{k}$ if and only if $y \in B$.

Proof. For convenience, we identify the space $\mathbb{N}^{N}$ with the irrational numbers in $[0,1]$ (see [1], Section 3). We fix three numbers $p, q$, and $r$ such that $a<p<q<r<b$. Let $K$ denote a nowhere dense perfect subset of $[a, p]$. As the set of bounded intervals contiguous to $K$ has order type $\eta$ (dense, unbordered, countable) there is a 1-1 order preserving mapping, $H$, from the binary fractions onto this set of intervals. If $x_{\tau}$ is a binary fraction, we let $J_{\tau}$ denote the open interval concentric with $H\left(x_{\tau}\right)$ but of half the length. Next we apply Lemma 6 for the Borel set $B$ and obtain the set

$$
M=\bigcup_{\tau}\left(\left\{x_{\tau}\right\} \times I_{\tau}\right)
$$

and the countable ordinal $\beta$ satisfying 1 . and 2 . of Lemma 6 . We define

$$
G_{1}=\bigcup\left(J_{\tau} \times I_{\imath}\right)
$$

where the union is taken over all finite sequences of natural numbers. Let $\left\{L_{\alpha}: \alpha<\beta \omega k\right\}$ be a discrete set of open subintervals of $[q, r]$ of order type $\beta \omega k$. Then we define

$$
G_{2}=\bigcup_{\alpha>\beta \omega k}\left(L_{\alpha} \times[0,1]\right)
$$

and

$$
G=G_{1} \cup G_{2}
$$

Now we define the map $T$ by (5.1). We define $F:[0,1] \rightarrow \mathscr{C}[0,1]$ by $F(y)(x)=(c-b) T(y)(x)+b$ for $y \in[0,1]$ and $x \in[a, b]$. We then set $F(y)(0)=0, F(y)(1)=1$ and complete the definition by insisting $F(y)$ be linear on each of the intervals $[0, a]$ and $[b, 1]$. It is evident from this definition that $F(y) \in \mathscr{N}$ for every $y \in[0,1]$ and it follows directly from Lemma 5 that $F$ is continuous at each irrational $y$. Finally, for each $y$, $C(F(y))=G^{y}=G_{1}^{y} \cup G_{2}^{y}$. The set of components for $G_{2}^{y}$ is precisely $\left\{L_{\alpha}: \alpha<\beta \omega k\right\}$ and has order type $\beta \omega k$. The nature of the components of $G_{1}^{y}$ depends on whether $y \in B$ or not and we consider these cases separately.

Case 1. $y \notin B$.

It follows from Lemma 6 that the components of $G_{1}^{y}$ contain a decreasing sequence of intervals converging, say, to $x^{*}$, and that every decreasing sequence of components converges to $x^{*}$. If $F(y) \in C^{k}$, then there are points $a=x_{0}<x_{1}<\cdots<x_{k}=b$ satisfying the 
conditions of Lemma 3. As $a \leqq x^{*}<b$ there is a unique $n \in\{0,1, \ldots, k-1\}$ such that $x^{*} \in\left[x_{n}, x_{n+1}\right)$. But then the components of $C\left(F(y) \mid\left[x_{n}, x_{n+1}\right)\right)$ are not well ordered and for each $i \neq n$ the components of $C\left(F(y) \mid\left[x_{i}, x_{i+1}\right)\right)$ are well ordered. Such a situation bodes ill for the homeomorphism guaranteed by Lemma 3 . This contradiction entails that if $y \notin B$ then $F(y) \notin C^{k}$.

\section{Case 2. $y \in B$.}

In this case, Lemma 6 yields that the components of $G_{1}^{y}$ are well ordered and of order type $\alpha<\beta$. As $\alpha+\beta \omega k=\beta \omega k$, the order type of $G_{1}^{y} \cup G_{2}^{y}$ is $\beta \omega k$. Further, as each set of components $G_{1}^{y}$ and $G_{2}^{y}$ is discrete and the two sets are separated by the interval $(p, q)$, the entire collection of components of $G^{y}$ is discrete. But then, Lemma 4 establishes the existence of the requisite points $a=x_{0}<x_{1}<\cdots<x_{k}=b$ and the increasing homeomorphism $\phi:\left[x_{0}, x_{k-1}\right] \rightarrow\left[x_{1}, x_{k}\right]$ which guarantee, via Lemma 3 , that $F(y) \in \mathscr{C}^{k}$. This completes the proof of Lemma 7.

As we saw in the introduction to this section Lemma 7 can now be used to prove the following theorem.

Theorem 8. Each of the sets $\mathscr{C}^{k}$ and $\mathscr{I} \cap \mathscr{C}^{k}$ is analytic and non-Borel in $\mathscr{C}[0,1]$ for $k=2,3, \ldots$

Remark 9. As we saw at the beginning of this section, Lemma 7 actually proves the slightly stronger result that $\mathscr{N} \cap \mathscr{C}^{k}$ is analytic and non-Borel in $\mathscr{C}[0,1]$ for $k=2,3, \ldots$.

Although this completes the proof of the main result of Section 1, there are some additional facts which we will need in the subsequent sections.

Proposition 10. If $f \in \mathcal{N}_{a b c}$, then for each $i=2,3, \ldots, f^{i} \in \mathcal{N}_{A_{i} b c_{i}}$ where $A_{i}=a^{i} / b^{i-1}$ and $C_{i}=1-(1-c)^{i} /(1-b)^{i-1}$.

Proof. For each $i=1,2,3, \ldots$ the facts that $f^{i}$ is increasing, $f^{i}(0)=0$, and $f^{i}(1)=1$ follow directly from the hypothesis that $f \in \mathcal{N}_{a b c}$. Further, as $f$ is linear and increasing on $[0, a]$ and $[b, 1]$ it follows that $f^{i}$ is linear on $\left[0, A_{i}\right]$ and $[b, 1]$. An easy computation shows that $f^{i}\left(A_{i}\right)=b$ and $f^{i}(b)=C_{i}$.

Proposition 11. Suppose $B \subset \mathbb{N}^{N}$ is Borel, $F$ is as in Lemma 7, and $y \notin B$. Then $F^{i}(y) \in \mathscr{C}^{j}$ if and only if $j$ divides $i$.

Proof. The sufficiency is obvious; for the necessity we again rely on the structure of the intervals of local constancy. As $y \notin B$, every decreasing sequence of components of $C(F(y))$ converges to the same real number. As $F(y) \in N_{a b c}, F(y)(a)=b, F(y)(b)=c$, and $F(y)$ is linear on each of the intervals $[0, a]$ and $[b, 1]$. From these it follows that any decreasing sequence of components of $C\left(F^{i}(y)\right)$ converges to one of exactly $i$ points, one 
in each of the intervals $[a, b),\left[A_{2}, a\right)$, and $\left[A_{n}, A_{n-1}\right) n=2,3, \ldots, i$. From Proposition 10 we know that $F^{i}(y) \in \mathcal{N}_{A_{i} b C_{i}}$. If $F^{i}(y) \in \mathscr{C}^{j}$ then these $i$ points must be equally distributed among the $j$ intervals guaranteed by Lemma 3 . This completes the proof of Proposition 11.

\section{Decreasing iterates of odd exponent}

In this section we show that if $k \geqq 3$ is odd and $\mathscr{D}$ denotes the set of decreasing functions in $\mathscr{C}$, then the set $\mathscr{D} \cap \mathscr{C}^{k}$ is analytic and non-Borel. The fact that $\mathscr{D} \cap \mathscr{C}^{k}$ is analytic is obvious as $\mathscr{D}$ is closed in $\mathscr{C}[0,1]$. Our method is to prove that for every odd $k \geqq 3$ and every Borel set $B \subset \mathbb{N}^{N}$ there exists a continuous map $W: \mathbb{N}^{N} \rightarrow \mathscr{D}$ such that if $y \in \mathbb{N}^{N}$ then $W(y) \in \mathscr{C}^{k}$ if and only if $y \in B$. As we saw in Section 1 , the existence of such a map proves that $\mathscr{D} \cap \mathscr{C}^{k}$ is non-Borel. We shall define $W$ as $\Phi \circ F$, where $\Phi$ maps a certain subclass of $\mathscr{C}$ (containing $\mathscr{N}$ ) into $\mathscr{D}$ and $F$ is the map found in Lemma 7 .

Let $\mathscr{M}$ denote the set of functions $f \in \mathscr{C}$ such that $f(1)=1$ and $f(x)<1$ for $x \in[0,1)$. For $f \in \mathscr{M}$ we define

$$
\Phi(f)(x)= \begin{cases}1-\frac{1}{2} f(2 x), & x \in\left[0, \frac{1}{2}\right] \\ \frac{1}{2} f(2-2 x), & x \in\left(\frac{1}{2}, 1\right]\end{cases}
$$

Lemma 12. The map $\Phi$ defined above has the following properties:

(i) $\Phi(f) \in \mathscr{C}$ for every $f \in \mathscr{M}$.

(ii) $\Phi(f) \in \mathscr{D}$ for every $f \in \mathscr{I} \cap \mathscr{M}$.

(iii) $\Phi(f) \circ \Phi(g)=1-\Phi(f \circ g)$ for every $f, g \in \mathscr{M}$.

(iv) $\Phi\left(\mathcal{N} \cap \mathscr{C}^{k}\right) \subset \mathscr{C}^{k}$ for every odd $k$.

(v) If $k$ is odd, then $f^{2} \in \mathscr{C}^{k}$ whenever $f \in \mathscr{M}$ and $\Phi(f) \in \mathscr{C}^{k}$.

Proof. Property (i) follows from the fact that $f(1)=1$ for every $f \in \mathscr{M}$ and (ii) is obvious from the definition of $\Phi$. An easy computation gives (iii). To prove (iv) let $k$ be odd and $f \in \mathscr{N} \cap \mathscr{C}^{k}$. From Lemma 2 we deduce that $f=g^{k}$ where $g \in \mathscr{I}, g(1)=1$ and $g$ is strictly increasing on $[b, 1]$. These imply that $g \in \mathscr{M}$. It now follows easily from (iii) and the fact that $k$ is odd that $\Phi(f)=\Phi\left(g^{k}\right)=(\Phi(g))^{k} \in \mathscr{C}^{k}$.

To prove (v) suppose that $f \in \mathscr{M}$ and $\Phi(f)=g^{k}$ where $g \in \mathscr{C}$. It follows from the definitions of $\mathscr{M}$ and $\Phi$ that $\Phi(f)$ has a unique fixed point at $x=\frac{1}{2}$ and that $\Phi(f)$ attains the value of $\frac{1}{2}$ only at $\frac{1}{2}$. Therefore $g$ has the same two properties. Consequently, either $g(x)<\frac{1}{2}$ for every $x \in\left[0, \frac{1}{2}\right)$ or $g(x)>\frac{1}{2}$ for every $x \in\left[0, \frac{1}{2}\right)$. The former is impossible since $\Phi(f)=g^{k}$ and $\Phi(f)(x)>\frac{1}{2}$ on $\left[0, \frac{1}{2}\right)$. Hence $g(x)>\frac{1}{2}$ on $\left[0, \frac{1}{2}\right)$ and the same argument shows that $g(x)<\frac{1}{2}$ on $\left(\frac{1}{2}, 1\right]$. This, together with the definition of $\Phi$, implies that there are functions $g_{1}, g_{2} \in \mathscr{M}$ such that $\Phi\left(g_{1}\right)\left|\left[0, \frac{1}{2}\right]=g\right|\left[0, \frac{1}{2}\right]$ and $\Phi\left(g_{2}\right)\left|\left[\frac{1}{2}, 1\right]=g\right|\left[\frac{1}{2}, 1\right]$. Then for $x \in\left[\frac{1}{2}, 1\right], g(x)=\Phi\left(g_{2}\right)(x) \in\left[0, \frac{1}{2}\right]$ and hence, $g^{2}(x)=\Phi\left(g_{1}\right) \circ \Phi\left(g_{2}\right)(x) \in\left[\frac{1}{2}, 1\right]$. This implies that 


$$
g^{2 k}(x)=\left(\Phi\left(g_{1}\right) \circ \Phi\left(g_{2}\right)\right)^{k}(x) \quad \text { for } \quad x \in\left[\frac{1}{2}, 1\right] .
$$

By (iii), $1-\Phi\left(f^{2}\right)=(\Phi(f))^{2}=g^{2 k}$. On the other hand, (iii) implies that whenever $m$ is even and $f_{1}, f_{2}, \ldots, f_{m} \in \mathscr{M}$, we have

$$
\Phi\left(f_{1}\right) \circ \Phi\left(f_{2}\right) \circ \cdots \circ \Phi\left(f_{m}\right)=1-\Phi\left(f_{1} \circ f_{2} \circ \cdots \circ f_{m}\right) .
$$

Hence

$$
\left(\Phi\left(g_{1}\right) \circ \Phi\left(g_{2}\right)\right)^{k}=1-\Phi\left(\left(g_{1} \circ g_{2}\right)^{k}\right)
$$

By (12.1), we have

$$
\Phi\left(f^{2}\right)\left|\left[\frac{1}{2}, 1\right]=\Phi\left(\left(g_{1} \circ g_{2}\right)^{k}\right)\right|\left[\frac{1}{2}, 1\right] .
$$

But, if $f_{1}, f_{2} \in \mathscr{M}$ and $\Phi\left(f_{1}\right)\left|\left[\frac{1}{2}, 1\right]=\Phi\left(f_{2}\right)\right|\left[\frac{1}{2}, 1\right]$ then $f_{1}=f_{2}$. Hence, it follows from (12.2) that $f^{2}=\left(g_{1} \circ g_{2}\right)^{k} \in \mathscr{C}^{k}$.

Lemma 13. For every Borel set $B \subset \mathbb{N}^{N}$ and odd $k \geqq 3$, there is a continuous map $W: \mathbb{N}^{N} \rightarrow \mathscr{D}$ such that $W(y) \in \mathscr{C}^{k}$ if and only if $y \in B$.

Proof. We put $W=\Phi \circ F$, where $\Phi$ is the mapping described above and $F$ is the function defined for the Borel set $B$ in Lemma 7. If $y \in B$ then, by Lemma 7, $F(y) \in \mathcal{N} \cap \mathscr{C}^{k}$ and hence $W(y)=\Phi(F(y)) \in \mathscr{D} \cap \mathscr{C}^{k}$ by (ii) and (iv) of Lemma 12. On the other hand, if $y \in \mathbb{N}^{N}$ and $W(y)=\Phi(F(y)) \in \mathscr{C}^{k}$ then, by (v) of Lemma $12,(F(y))^{2} \in \mathscr{C}^{k}$. But 2 does not divide $k$ and hence Proposition 11 implies that $y \in B$.

As we saw at the beginning of this section, Lemma 13 establishes the following result.

Theorem 14. If $k \geqq 3$ is odd, then $\mathscr{D} \cap \mathscr{C}^{k}$ is analytic and non-Borel in $\mathscr{C}[0,1]$.

\section{Decreasing iterates of even exponent}

Our goal in this section is to prove the following characterization of the class $\mathscr{D} \cap \mathscr{C}^{k}$, $k$ even.

Theorem 15. For each even $k, \mathscr{D} \cap \mathscr{C}^{k}=\mathscr{D} \cap \mathscr{C}^{2}$. Moreover, if $f \in \mathscr{D}$ and $k$ is even then $f \in \mathscr{C}^{\mathrm{k}}$ if and only if $C(f)$ contains the range of $f$.

From this we can immediately infer that for even $k, \mathscr{D} \cap \mathscr{C}^{k}$ is Borel and indeed, is $F_{\sigma}$.

Corollary 16. For every even $k, \mathscr{D} \cap \mathscr{C}^{k}$ is an $F_{\sigma}$ subset of $\mathscr{C}[0,1]$.

Proof. It is easy to see that if $p<q<r<s$ then the set of functions $f \in \mathscr{D}$ such that $f$ 
is constant on $[p, s] \cap[0,1]$ and the range of $f$ is contained in $[q, r]$ is closed in $\mathscr{C}[0,1]$. By Theorem $15, \mathscr{D} \cap \mathscr{C}^{k}$ is the union of all such sets where $p, q, r$, and $s$ are rational.

We turn now to the proof of Theorem 15 which is accomplished via a series of results.

Theorem 17. If $f \in \mathscr{C}$ and $C(f)$ contains the range of $f$, then $f \in \mathscr{C}^{k}$ for every $k=1,2, \ldots$

Proof. The range of $f$ is a closed interval while $C(f)$ is relatively open in $[0,1]$. Let $I$ denote the component of $C(f)$ containing the range of $f$ and set $u \equiv f \mid I$. If $I=[0,1], f$ is constant on $I$ and the conclusion follows as $f=f^{k}$. Therefore we may assume $I \neq[0,1]$, and we first assume $I=(a, b)$ where $0<a<b<1$. If $m=\min \{f(x): x \in[0,1]\}$ and $M=\max \{f(x): x \in[0,1]\}$ then the hypothesis implies $a<m \leqq u \leqq M<b$.

Let $k \geqq 2$ be fixed, and choose points $x_{i}, i=1,2, \ldots, k$ such that $0=x_{1}<a<x_{2}<\cdots<$ $x_{k}<m$. We define the function $g$ to be the increasing linear map from $\left[x_{i-1}, x_{i}\right]$ onto $\left[\mathrm{x}_{\mathrm{i}}, x_{i+1}\right], i=2,3, \ldots, k-1$. Then $g^{k-2}$ maps $\left[0, x_{2}\right]$ onto $\left[x_{k-1}, x_{k}\right]$. Let

$$
c=g^{k-2}(a) \in\left(x_{k-1}, x_{k}\right) \text {. }
$$

Define $g$ to be linear and increasing on each of the intervals $\left[x_{k-1}, c\right]$ and $\left[c, x_{k}\right]$, mapping them respectively onto $\left[x_{k}, m\right]$ and onto $[m, f(0)]$. Next define

$$
\begin{aligned}
& g(x)=f\left(\frac{a}{m-x_{k}}\left(x-x_{k}\right)\right) \text { if } x \in\left[x_{k}, m\right], \text { and } \\
& g(x)=u \quad \text { if } \quad x \in[m, u] .
\end{aligned}
$$

At this point $g$ has been defined on $[0, u]$ and it is easy to check that $g$ is continuous here using the fact that $g(m)=f(a)=u$. The definition of $g$ on $[u, 1]$ is analogous but using $M$ and $b$ in place of $m$ and $a$ respectively.

We prove that $f=g^{k}$. Since $g^{k-1}$ maps $[0, a]$ linearly onto $\left[x_{k}, m\right]$, we have, for $x \in[0, a]$,

$$
g^{k-1}(x)=\frac{m-x_{k}}{a} x+x_{k}
$$

Therefore, by the definition of $g$ in $\left[x_{k}, m\right]$ we deduce that $g^{k}(x)=f(x)$ for $x \in[0, a]$. Further, since $g^{k-1}$ maps $\left[a, x_{2}\right]$ into $[m, f(0)] \subset[m, M]$ and $g([m, M])=\{u\}$, we have $g^{k}(x)=u=f(x)$ whenever $x \in\left[a, x_{2}\right]$. Since $g\left(\left[x_{k-1}, x_{k}\right]\right)=\left[x_{k}, f(0)\right]$ and

$$
g\left(\left[x_{k}, f(0)\right]\right)=g\left(\left[x_{k}, m\right]\right) \cup g([m, f(0)]) \subset[m, M] \cup g([m, M])=[m, M] \cup\{u\}=[m, M],
$$

we have $g^{3}\left(\left[x_{k-1}, x_{k}\right]\right)=\{u\}$. Therefore, if $3 \leqq i \leqq k$ then 


$$
g^{k}\left(\left[x_{i-1}, x_{i}\right]\right)=g^{i}\left(\left[x_{k-1}, x_{k}\right]\right)=g^{i-3}(\{u\})=\{u\} .
$$

Since $f(x)=u$ for $x \in\left[x_{2}, x_{k}\right]$, this proves that $f(x)=g^{k}(x)$ on $\left[x_{2}, x_{k}\right]$. If $x \in\left[x_{k}, u\right]$, then $g(x) \in[m, M]$ so that $g^{2}(x)=g^{k}(x)=u=f(x)$. The same argument applies if $x \in[u, 1]$ and, as such, the proof that $f \in \mathscr{C}^{k}$ is complete.

Next consider the case $I=(a, 1]$. Then for each $k$, the function $g$ is defined as above on the interval $[0, u]$ but is defined to be the constant $u$ on $[u, 1]$. The proof that $f(x)=g^{k}(x)$ for $x \in[0, u]$ is exactly as that given above while the fact that $g^{k}(x)=f(x)$ for $x \in[u, 1]$ now follows from the fact that $f(x)=g(x)=u$ on $[u, 1]$.

The case $I=[0, b)$ is analogous and this completes the proof of Theorem 17.

Lemma 18. Let $f \in \mathscr{D} \cap \mathscr{C}^{2}$ and let $u$ denote the (unique) fixed point of $f$. Then $f(x)=u$ holds whenever $x \in[f(1), f(0)]$.

Proof. Let $f^{-1}(\{u\})=[\alpha, \beta]$; we must prove that $\alpha \leqq f(1)$ and $\beta \geqq f(0)$. Suppose this is not true and assume, for example, that $f(1)<\alpha$. Let $f=g^{2}, g \in \mathscr{C}$. Since $u$ is the sole fixed point of $f, u$ is also the only fixed point of $g$. As $f=g^{2}$ is decreasing, this implies that $g(x)>x$ for $x<u$ and $g(x)<x$ for $x>u$. Set $g(1)=w$.

As a first case, suppose $w>\beta$. Then $g(w)=f(1) \leqq u$ and $g(1)=w>\beta \geqq u$. Hence there is a $y \in[w, 1]$ such that $g(y)=u$. Then $f(y)=g^{2}(y)=g(u)=u$ which is impossible as $y \geqq w>\beta$.

Next, suppose $w<\alpha$. Then $g(g(w))=f(w)>u$ and $g(w)=f(1) \leqq u$. Hence, there is a $y \in[w, g(w)]$ with $g(y)=u$. Again, $f(y)=g(u)=u$ which is impossible since $y \leqq g(w)=f(1)<\alpha$.

Therefore, we may suppose $w \in[\alpha, \beta]$ and hence that $f(w)=u$. Now, $f^{2}(1)=g^{4}(1)=$ $g^{3}(w)=g(f(w))=g(u)=u$ which again is impossible as $f(1)<\alpha$. This final contradiction completes the proof of Lemma 18.

We now turn to the proof of Theorem 15.

Proof of Theorem 15. Let $f \in \mathscr{D}$. If $C(f)$ contains the range of $f$ then, by Theorem 17, $f \in D \cap C^{k}$ for every $k$. If $f \in \mathscr{D} \cap \mathscr{C}^{k}$ with $k$ even then, obviously, $f \in \mathscr{D} \cap \mathscr{C}^{2}$ so that, by Lemma $18, f$ is constant on the interval $[f(1), f(0)]$. To complete the proof of Theorem 15 we must show that there is an $\varepsilon>0$ such that $f$ is constant on the interval $[f(1)-\varepsilon, f(0)+\varepsilon] \cap[0,1]$. As in Lemma 18 we let $u$ denote the only fixed point of $f$, let $[\alpha, \beta]=f^{-1}(\{u\})$, and let $g \in \mathscr{C}$ be such that $g^{2}=f$. We must show that

(i) either $\beta=1$ or $f(0)<\beta$ and

(ii) either $\alpha=0$ or $f(1)>\alpha$.

Suppose, for example, that (i) is false, that is, $f(0)=\beta<1$. We prove that this implies that $g$ is not constant on $[u, f(0)]$.

First we show $f(0)>u$. Indeed, if $u=f(0)=\beta$, then $f(z)=u$ for every $z \in[0, u]$. Hence either $g \equiv u$ in $[0, u]$ or $g([0, u])$ contains a one sided neighbourhood of $u$ on which $g \equiv u$. In each of these cases, $g \equiv u$ in a one sided neighbourhood of $u$. If this is a right 
neighbourhood then $f \equiv u$ in that neighbourhood which is impossible since $f(0)=u=\beta$. We conclude that $g$ is not constant in $[u, 1]$ so that $g([u, 1])$ contains a one sided neighbourhood of $u$ and $g<u$ in this neighbourhood. Thus, there is a $\delta>0$ such that $g(z)<u$ for $z \in(u, u+\delta)$ which again is a contradiction. Hence, $f(0)=\beta>u$.

Suppose $g$ is constant on $[u, f(0)]$. Then $g \equiv u$ on $[u, f(0)]$. Further, since $f(0)=\beta$, $g^{2}(z)=f(z)<u$ for $z \in(f(0), 1]$. But this entails that $g$ is not constant on $(f(0), 1]$ else this constant would be $g(f(0))=u$ which would imply that $g^{2}(z)=u$ on $(f(0), 1]$. Thus, $g((f(0), 1])$ contains a one sided neighbourhood of $u$ and $g<u$ on this neighbourhood. Since $g \equiv u$ on $[u, f(0)]$ there is a $\delta>0$ such that $g(z)<u$ for $z \in(u-\delta, u)$. Then there is an $\eta>0$ such that $f(z)=g^{2}(z)<u$ for $z \in(u-\eta, u)$ which is not the case. Hence $g$ cannot be constant on $[u, f(0)]$ as we claimed.

Now, set $v=g(0)$ so that $g(v)=f(0)$. We consider three cases.

Case 1. $v>f(0)$.

In this case $f(v)<u, g(f(0))=f(v)<u$, and $g(v)=f(0) \geqq u$. Hence, there is a $y \in(f(0), v]$ with $g(y)=u$. But then $f(y)=u$ which is impossible as $y>f(0)=\beta$.

Case 2. $f(1) \leqq v \leqq f(0)$.

Here we have $f(v)=u$ and $f(g(0))=g(f(0))=u$. As $g(v)=f(0)$, it follows that $g([v, f(0)]) \supset[u, f(0)]$. But $f \equiv u$ on $[v, f(0)]$ and hence $g$ is constant on $[u, f(0)]$ which, as we saw above, is impossible.

Case 3. $v<f(1)$.

In this case, $g(v)=f(0) \geqq u$ and $g(0)=v<u$ so that there is a $y \in[0, v]$ such that $g(y)=u$. Then $f(y)=u$ and hence, as $y \leqq v<f(1), f(v)=u$. Therefore $f \equiv u$ on $[v, f(0)]$. But $g([v, f(0)]) \supset[u, f(0)]$ and we again conclude that $g$ is constant on $[u, f(0)]$. This final contradiction completes the proof.

\section{REFERENCE}

1. K. Kuratowski, Topology I (Academic Press, 1966).

Department of Mathematics

St. Olaf College

Northfield, MN, U.S.A.

Department of Analysis

EOUTVÖS LORÁND UNIVERSITY

BudapFst, Hungary 